\title{
Prevalencia y factores de riesgo asociados a la adquisición de Acinetobacter baumannii Multidrogoresistente en el servicio de Microbiología del Hospital Escuela durante el periodo de Enero a Septiembre de 2012
}

Tesina como requisito de graduación para el título de Dra. En Microbiología con orientación en análisis clínicos

Autor: Dra. Blanca Hernández

Asesor: Dra. Milena Vanegas

Afiliaciones: Hospital Escuela Universitario, Servicio de microbiología. Tegucigalpa, Honduras.

\section{Resumen:}

Justificación: Acinetobacter baumannii es un bacilo o cocobacilo Gram negativo, aerobio y no fermentador de la glucosa, que se aísla mayoritariamente en pacientes hospitalarios, de. Su alta resistencia a los antibióticos lo vuelve un patógeno de difícil erradicación en los hospitales, convirtiéndose así en un nosocomial de gran importancia en la mayoría de hospitales alrededor del mundo. Objetivo: Determinar la prevalencia y los factores de riesgo asociados a la adquisición de Acinetobacter baumannii Multidrogorresistentes en el Servicio de Microbiología del Hospital Escuela durante los meses de Enero a Septiembre del 2012. Métodos: Se realizó un estudio de tipo descriptivo transversal en todos los cultivos positivos por Acinetobacter baumannii recibidos en el servicio de microbiología del Hospital Escuela durante el período de Enero a Septiembre del 2012. Resultados: Se encontró una prevalencia de $65.2 \%$ cuyos factores de riesgo con mayor asociación fueron: antibióticoterapia previa $(87 \%)$, enfermedad subyacente grave $(77 \%)$, uso de catéter $(30 \%)$, ventilación mecánica (18\%), cirugía (20\%) y el uso de sonda (4\%). De los pacientes encontrados con hasta 3 factores de riesgo simultáneamente, el 71\% desarrolló una infección. Conclusiones: La prevalencia es bastante alta, sin embargo es comparable a la situación a nivel mundial, su principal asociación es debido al uso de antibióticoterapia y estados de inmunosupresión, así como el uso de técnicas invasivas sin los cuidados de asepsia necesarios.

\section{Correspondencia:}

Favor dirigir correspondencia a

Autor: Dra. Blanca Alejandra Hernández

Teléfono: (+504)9842-7490

Correo electrónico: blancahp9@hotmail.com 